\title{
Monitoring of Cutaneous Adverse Drug Reactions in a Tertiary Care Hospital
}

Raja Amrinder ${ }^{1 *}$, Inderpal Kaur, Jatinder Singh and Tejinder Kaur ${ }^{2}$

${ }^{1}$ Department of Pharmacology, Government Medical College, Amritsar, Punjab, India

${ }^{2}$ Department of Dermatology, Venereology and Leprosy, Government Medical College, Amritsar, Punjab, India

\begin{abstract}
Introduction: Cutaneous adverse drug reactions (ADRs) are the most frequently occurring ADRs to drugs. These reactions have a varied morphology and are responsible for significant mortality and morbidity. The aim of this study is to characterize the morphological patterns of cutaneous ADRs and to determine the incriminating agents.

Method: The study was carried out in the Dermatology, Venereology and Leprosy department, Guru Nanak Dev Hospital, Government Medical College, Amritsar from $1^{\text {st }}$ March 2014 to $31^{\text {st }}$ May 2015. World Health OrganizationUppsala monitoring centre (WHO-UMC) causality scale was used to determine the causality of cutaneous ADRs and severity was assessed using Hartwigs severity scale.

Results: In the present study, the highest incidence of cutaneous ADRs was in the age group of 31-40 years $(25.0 \%)$, and more frequently in female patients $(54.2 \%)$. Antimicrobials were the most commonly implicated drugs (37.5\%) followed by Non-Steroidal Anti-inflammatory drugs (NSAIDs) $(25.0 \%)$, various combination drugs $(10.0 \%)$, corticosteroids and antiepileptics (6.6\%). The most commonly observed morphological pattern was of Fixed drug eruptions $(33.3 \%)$ followed by maculopapular rash $(30.8 \%)$ and Steven Johnson Syndrome $(5.8 \%)$. Causality assessment was certain, probable and possible for $1.6 \%, 93.3 \%$ and $41.5 \%$ of the reactions, respectively. 109 cases were of level 3 severities, 10 cases to level 4 severities and one case of level 7 where ADR was responsible for death in one patient.

Discussion: Most of the adverse drug reactions are preventable, provided the drugs are used rationally. Antimicrobials were the most common causative group and fixed drug eruption was the most frequently encountered morphological pattern. Therefore it is imperative that in each patient the risk of drug administration should be weighed against the expected therapeutic benefit.
\end{abstract}

Keywords: Cutaneous adverse drug reactions; Drug eruptions; Morphological patterns; Causality assessment

\section{Introduction}

Drugs are prescribed with an intention of relieving suffering but sometimes they themselves can cause adverse drug reactions ranging from minor inconvenience to serious organ dysfunction, or even death. Their awareness to the medical world, public and official bodies was highlighted mainly after thalidomide disaster in 1961and since then several worldwide studies have shown ADRs to be a major cause of morbidity and mortality [1]. While the exact epidemiology remains to be assessed in India, ADRs have recently emerged as leading killers [2].

WHO defines ADRs as 'any response to a drug which is noxious, unintended and which occur at doses normally used in man for prophylaxis, diagnosis, or therapy of diseases, or for the modification of physiological function' [3].

Clinically important ADRs are diverse but cutaneous ADRs are most common among the various adverse reactions and attributed by the drugs [4]. Cutaneous ADRs occur in up to $8 \%$ of global population and in $2-3 \%$ of hospitalized patients [5]. The incidence of cutaneous ADRs in developed countries has been found as $1-3 \%$ with comparatively higher incidence between $2 \%$ and $5 \%$ in developing countries.

Different studies show variation in the data based on presentation of cutaneous drug reaction, its distribution amongst both sexes, the offending drug and causality assessment. Due to these wide divergences, it is necessary to generate ADR data, categorizing the type and severity of reaction and the discriminating drug along with causality assessment.

\section{Aims and Objectives}

- To characterize the clinical spectrums of cutaneous ADRs and to determine the incriminating drugs.

- To access the causality and severity of cutaneous ADRs.

\section{Methods}

A prospective, observational, questionnaire based study was undertaken in the Department of Dermatology, Venereology and Leprosy, Guru Nanak Dev Hospital, a 1000 bedded tertiary care teaching hospital from $1^{\text {st }}$ March 2014-31 ${ }^{\text {st }}$ May 2015. All new patients irrespective of age and sex, having various cutaneous eruptions attending the outpatient clinic and admitted to ward of Dermatology, Venereology and Leprosy department directly and patients with cutaneous eruptions referred from inpatient or outpatient departments of other specialties during the study period were screened for suspected cutaneous ADRs and only those satisfying the inclusion criteria were enrolled. The study protocol was approved by Institutional

*Corresponding author: Raja Amrinder, Department of Pharmacology, Government Medical College, Circular road, Amritsar-143001, Punjab, India, Tel: 9876626481; E-mail: dramrinder19@gmail.com

Received: April 11, 2016; Accepted May 04, 2016; Published May 09, 2016

Citation: Amrinder R, Kaur I, Singh J, Kaur T (2016) Monitoring of Cutaneous Adverse Drug Reactions in a Tertiary Care Hospital. J Pharmacovigilance 4: 207. doi:10.4172/2329-6887.1000207

Copyright: (c) 2016 Amrinder R, et al. This is an open-access article distributed under the terms of the Creative Commons Attribution License, which permits unrestricted use, distribution, and reproduction in any medium, provided the original author and source are credited. 
Ethics Committee. Written Informed consent was taken from all the participants. The data was compiled based on a suitable study design. To establish the etiologic agent for a particular type of reaction, attention was paid to the detailed drug history, temporal correlation with the drug, duration of the rash, approximate incubation period, morphology of the cutaneous eruption, and improvement of lesions after withdrawal of drug, i.e., Dechallenge (wherever possible). Rechallenge of the offending drugs was not done. The diagnosis of drug reaction was clinical. However, skin biopsy for histopathological examination was done only in specific cases to confirm drug reaction, when differentiation between the idiopathic from the drug-induced reaction was needed. After establishing the clinical diagnosis of ADR, appropriate treatment was given by the consultant dermatologist after taking into account the age of the patient, severity of lesion and medical history of the patient. Hospitalization was done for severe cases. The follow up of the patient varied depending on severity of the reaction.

The results were calculated in form of percentages. Cutaneous ADR patterns, drug classes accounting for ADRs and the offending drugs were categorized in this study. The causality relationship was assessed as per WHO-UMC causality scale, which classified the reactions as certain/definite, probable/likely and possible. The unlikely, conditional/ unclassified and unassessable/unclassifiable reactions were excluded. The cutaneous ADR's were categorized into mild, moderate and severe reactions according to Hartwig severity scale.

\section{Results}

A total of 120 patients with a definite history of drug intake were enrolled in the present study. Majority of cases, i.e., 30 (25.0\%) were in the age group of 31-40 years followed by 26 (21.0\%) in age group of 21-30 years and the lowest number of three $(2.5 \%)$ cases in the age group $>70$ years. The youngest patient was 3.5 years old and the oldest was 75 years of age. In the present study, females (54.2\%) were more frequently affected than males (45.8\%). Out of 120 patients with cutaneous ADRs, $45(37.5 \%)$ patients had a positive history of previous cutaneous adverse drug reactions. Except a few cases which showed a minor increase in severity of recent cutaneous lesions, most of the positive cases presented with the lesions of same severity as the previous ones. $34(28.3 \%)$ patients had a positive history of allergic disorders like atopic dermatitis, contact dermatitis, allergic rhinitis, bronchial asthma and urticaria. Only five (4.2\%) patients showed positive family history. The drug classes that were associated with a risk of genetic predisposition of causing cutaneous ADRs included fluoroquinolones [ofloxacin (one case)], aminopenicillins [amoxicillin (one case)], antigout drugs [allopurinol (one case)] and antifungal drugs [fluconazole (two cases)]. FDE was the most frequently observed morphological pattern in $40(33.3 \%)$ patients, followed by maculopaular rash in 37 (30.8\%) patients and SJS in seven (5.8\%) (Figure 1). The most common drug groups implicated in cutaneous ADRs included antimicrobials in $45(37.5 \%)$ patients followed by NSAIDs in $30(25.0 \%)$ patients, various combination drugs and corticosteroids each in eight (6.6\%) patients (Figure 2). Among antimicrobials, the most common incriminating drug class was of antibiotics in 26 (57.7\%) patients, with maximum number of cutaneous ADRs, i.e., 9 from fluoroquinolone group (Figure 3 ). The antibiotic most frequently implicated to cause cutaneous ADRs was ampicillin in five patients (Figure 4 and Table 1).

Among NSAIDs, out of 30 patients the majority of cutaneous ADRs were caused by propionic acid derivate, ibuprofen in 12 (40.0\%) patients; followed by a para-aminophenol derivate, paracetamol in $11(36.6 \%)$ and the acetic acid derivate, diclofenac in three (10.0\%) patients. A total of 12 patients showed cutaneous ADRs with various combinations of drugs from different groups. Five (41.6\%) patients with lamivudine+zidovu dine+nevirapine, three (25.0\%) patients with paracetamol+ibuprofen, two $(16.6 \%)$ patients with sulfamethoxazole+trimethoprim, one $(8.3 \%)$ patient each with ofloxacin+ornidazole and amlodipine+atenolol combination. Amongst corticosteroids four (50.0\%) patients showed cutaneous ADRs with betamethasone [inhaled beclomethasone one patient and topical in three patients], two (25.0\%) patients with topical application of mometasone furoate and two (25.0\%) patients with topical application of hydrocortisone. Antiepileptic drugs caused cutaneous ADRs in eight patients, with carbamazepine and phenytoin each in three (37.5\%) patients. Phenobarbitone and clonazepam caused ADRs each in one (12.5\%) patient. Hypolipidemic drugs (Niacin) and herbal drug were also found to cause certain cutaneous ADRs (Tables 1 and 2). The correlation of suspected incriminating drugs $v / s$ clinical

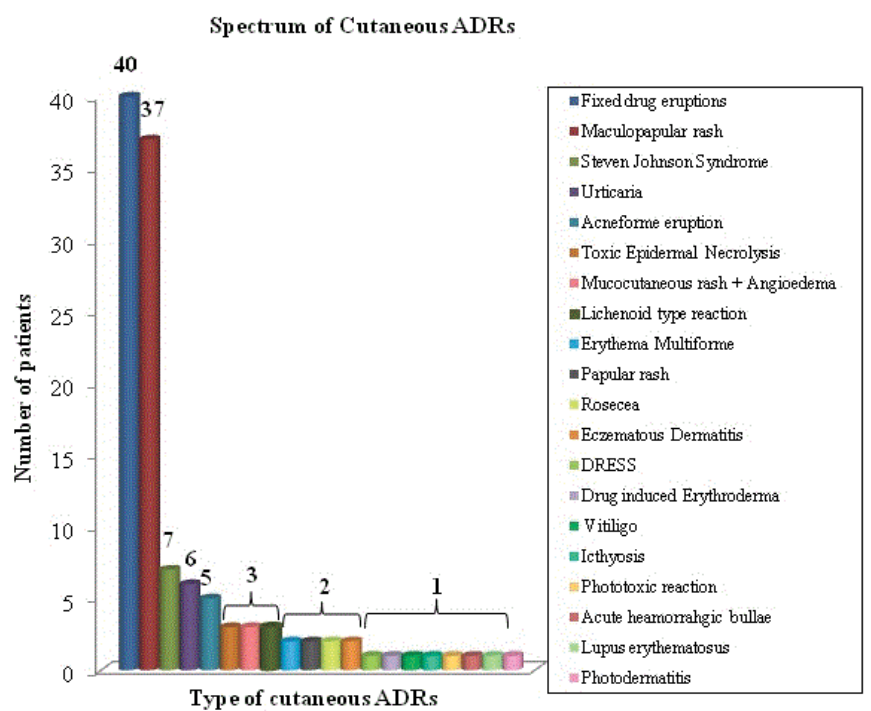

Figure 1: Spectrum of cutaneous ADRs.

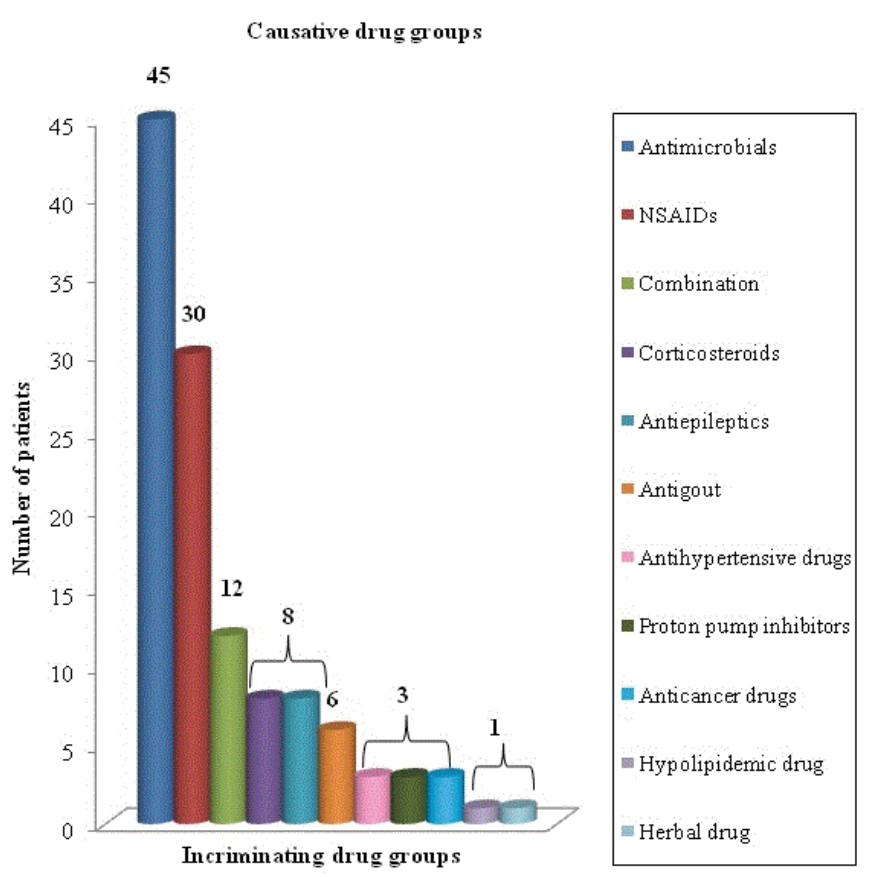

Figure 2: Causative drug groups. 


\begin{tabular}{|c|c|c|c|}
\hline S. No. & Incriminating drug class & $\begin{array}{l}\text { Number of patients } \\
\text { (n) }\end{array}$ & Incriminating drugs \\
\hline A. & Antimicrobials & 45 & \\
\hline 1 & Antibiotics & 26 & $\begin{array}{l}\text { Ofloxacin (4), Ciprofloxacin (4), Levofloxacin (1), Ampicillin (5), Amoxicillin (1), } \\
\text { Doxycycline (4), Cefprozil (1), Cefuroxime (1), } \\
\text { Cefixime (1), Azithromycin (2), Pyrazinamide (1), Rifampicin (1) }\end{array}$ \\
\hline II & Antiretrovirals & 12 & Efavirenz (7), Neverapine (4), Zidovudine (1) \\
\hline III & Antifungals & 3 & Fluconazole (3) \\
\hline IV & Antihelminthics & 2 & Ornidazole (1), Tinidazole (1) \\
\hline $\mathrm{V}$ & Antiprotozoal & 2 & Metronidazole (2) \\
\hline B. & NSAIDs & 30 & $\begin{array}{l}\text { Ibuprofen (12), Paracetamol (11), Diclofenac (3), Nimesulide (2), Aspirin (1), } \\
\text { Mefenamic acid (1) }\end{array}$ \\
\hline C. & Combination drugs & 12 & $\begin{array}{c}\text { Lamivudine+Zidovudine+Nevirapine (5), Paracetamol+lbuprofen } \\
\text { (3), Sulfamethoxazole+Trimethoprim (2), Ofloxacin+Ornidazole (1), } \\
\text { Amlodipine+Atenolol (1) }\end{array}$ \\
\hline D. & Corticosteroids & 8 & Betamethasone (4), Mometasone furoate (2), Hydrocortisone (2) \\
\hline E. & Antiepileptics & 8 & Carbamazepine (3), Phenytoin (3), Phenobarbitone (1), Clonazepam(1) \\
\hline F. & Antigout & 6 & Allopurinol (4), Colchicine (2) \\
\hline G. & Antihypertensives & 3 & Atenolol (2), Ramipril (1) \\
\hline H. & Proton pump inhibitors & 3 & Pantoprazole (3), Omeprazole (1) \\
\hline I. & Anticancer agents & 3 & Carboplatin (1), Cyclophosphamide (1), Rituximab (1) \\
\hline J. & Others & 2 & Niacin (1), Herbal drug (1) \\
\hline
\end{tabular}

Table 1: Suspected incriminating drugs in 120 patients of cutaneous ADRs.

\begin{tabular}{|c|c|c|c|}
\hline S. No. & Incriminating drug groups & \begin{tabular}{c} 
Clinical patterns \\
\hline 1
\end{tabular} & FDE (11), MP (19), SJS (3), Urticaria (3), MC+A (2), ERYM (2), ED (2), \\
PTR (1), LE (1), PD (1)
\end{tabular}

FDE, Fixed Drug Eruption; MP, Maculopapular Rash; SJS, Seven Johnson Syndrome; AEP, Acneiform Eruption; TEN, Toxic Epidermal Necrolysis; MC+A, Mucocutaneous Rash+Angioedema; ERYM, Erythema Multiforme; PR, Papular Rash; LTE, Lichenoid Type Reaction; ED, Eczematous Dermatitis; DRESS, Drug Rash With Eosinophilia And Systemic Symptoms; DIE, Drug Induced Erythroderma; PTR, Phototoxic Reaction; ABH, Angina Bullosa Heamorrahgica; LE, Lupus Erythematosus; PD, Photodermatitis

Table 2: Correlation of suspected incriminating drug groups $v / s$ clinical patterns in 120 patients of cutaneous ADRs.

Classes of Antibiotics causing cutaneous ADRs

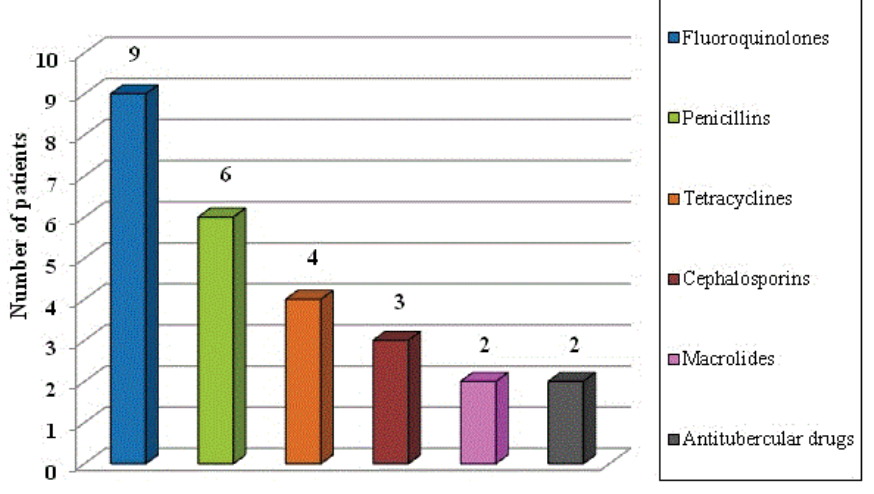

Incriminating drug classes

Figure 3: Classes of antibiotics causing cutaneous ADRs. patterns in 120 patients of cutaneous ADRs is shown in Table 2. The maximum cutaneous ADRs, i.e., 57 (47.5\%) occurred in the lag period of 2-14 days followed by $51(42.5 \%)$ in less than 2 days, $18(6.6 \%)$ in 15-60 days and only six (3.3\%) occurred in a lag period greater than 2 months. After the causality assessment of cutaneous ADRs as per WHO-UMC causality scale, majority of cases, i.e., 112 (93.3\%) were categorized as probable, six (5.0\%) cases as possible and only two (1.6\%) cases as certain. As per Hartwig severity scale, 109 cases were of level 3 severity where the treatment with the suspected drug was held, 10 patients belonged to level 4 where the treatment with the suspected drug was held and ADR was the cause of admission and one case of level 7 where ADR was responsible for death in one patient.

\section{Discussion}

In the present study 120 patients with cutaneous ADRs were studied with the maximum number of patients from the age group of 31-40 years. These findings were in concordance with other studies done by Balpande [6] and Anjaneyan [7]. This could be due to increased use of medications, drug-drug interactions and physiological alterations in them. The females in our study outnumbered the males. Mahapatra [8] and Lamani [9] also showed female preponderance in their studies. 
Antibiotics causing cutaneous ADRs

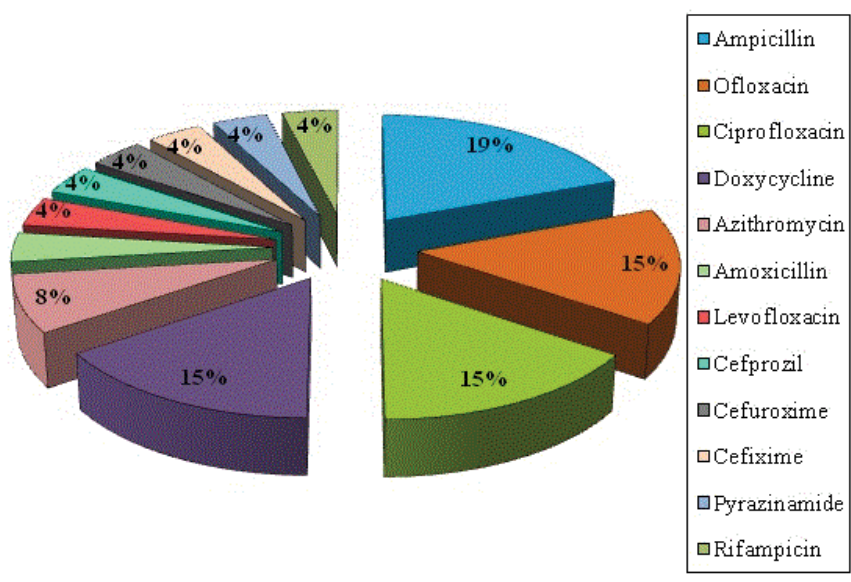

Figure 4: Antibiotics causing cutaneous ADRs.

The frequent use of over the counter drugs by females to alleviate pain in cases of headache and dysmenorrhea along with higher levels of endogenous hormones (like estradiol) can be contributing factors. 45 (37.5\%) patients had a previous history of cutaneous ADRs to drugs. Bai [10] reported a past history of ADRs in six (10\%) patients. Positive history of allergic disorders like atopic dermatitis, contact dermatitis, allergic rhinitis, bronchial asthma and urticaria was noticed in 34 (28.3\%) patients.

The most common cutaneous ADRs recorded were fixed drug eruptions (33.3\%), followed by maculopapular rash (30.8\%) and Steven Johnson syndrome $(5.83 \%)$. The most common incriminating drug class was of antimicrobials (37.5\%) and NSAIDs (25.0\%). The maximum numbers of cutaneous ADRs were induced by fluoroquinolone class. Our findings here are in accordance with a study done by Verma [11]. In present study Ampicillin was the most common culprit drug and was responsible for reactions like maculopapular rash, urticaria and eczematous dermatitis. Maculopapular rash was most commonly reported due to amoxicillin in study done by Sharma [12]. In the present study maximum numbers of cases were seen within lag period of 2-14 days. Maheswary [13] reported similar findings. After Causality assessment using WHO-UMC causality criteria, the implicated drug was found to be a probable cause in $93.3 \%$ of patients, possible in $5.0 \%$ of patients and certain in $1.6 \%$ of patients. Chowdhury (2012) found that the majority of cases were either probable $(41.5 \%)$ or possible $(39.6 \%)$ [14]. The assessment might vary with the type of scales/ algorithms used for the assessment of ADRs in different regions. Out of total 120 cases of cutaneous ADRs, maximum cases, i.e., 109 were of level 3 severities, 10 cases of level 4 and one case of level 7 severities.

\section{Conclusion}

Our study screened a number of subjects and a corresponding wide spectrum of commonly used drugs. However, there were also some limitations. Causality assessment might be uncertain especially as rechallenge was not attempted due to ethical reasons. There may be disparity between the observed and actual incidence of cutaneous ADRs due to underreporting of minor reactions. Hence, the practitioners and patients should be motivated to report any untoward incidence occurring with the drug. Continuous vigilance on all the drugs including traditional medicines is thus mandatory.

\section{References}

1. Deepa A (2012) Phramacovigilance-An Industry Prospective. Navi Mumbai Pharma publisher.

2. Patidar D, Rajput SM, Nirmal PN, Wenny S (2013) Implementation and evaluation of adverse drug reaction monitoring system in a tertiary care teaching hospital in Mumbai, India. Interdiscip Toxcicol 6: 41-46.

3. Srinivasan R, Ramya G (2011) Adverse drug reaction-Causality assessment IJRPC 1: 606-612.

4. Ghosh S, Leelavathi DA, Rao PM (2006) Study and evaluation of various cutaneous adverse drug reactions in Kasturba Hospital, Manipal. Indian Journal of Pharmaceutical Sciences 68: 212-215.

5. Bigby M (2001) Rates of cutaneous reactions to drugs. Arch Dermatol 137:765770 .

6. Balpande KG, Borkar AS, Badwaik RT (2013) Study of clinical patterns in patients with cutaneous adverse drug reactions. IJMPS 3: 34-39.

7. Anjaneyan G, Gupta R, Vora R (2013) Clinical study of adverse cutaneous drug reactions at a rural based tertiary care centre in Gujarat. National Journal of Physiology, Pharmacy and Pharmacology 3: 129-136.

8. Mahapatra S, Keshri PdU (2012) Adverse cutaneous drug reactions in a tertiary care centre patients: a prospective analysis. Journal of Applied Pharmaceutical Science 2: 96-98.

9. Lamani VL, Ratnakar JS, Kotinatot BC, Bhusan A (2015) Study of cutaneous adverse drug reactions in a tertiary care teaching hospital. International Journal of Basic and Applied Medical Sciences 5: 71-74.

10. Bai CK, Padma L, Mohan NTM, Veena DR (2014) Drug induced cutaneous adverse drug reactions in dermatology in Dr. B.R. Ambedkar Medical College. Int J Basic Clin Pharmacol 3: 706-710.

11. Verma R, Tiwari S, Gupta CM, Verma N (2014). Cutaneous adverse drug reactions-A study of clinical patterns, causality, severity and preventability. Journal of Dental and Medical Sciences 13: 102-109.

12. Sharma R, Dogra D, Dogra N (2015). A study of cutaneous adverse drug reactions at a tertiary center in Jammu, India. Indian Dermatol Online J 6: 168171.

13. Maheswary R, Singh J, Rai J, Puri KJPS (2006). Prospective study of adverse drug reactions manifesting as skin lesions in tertiary care hospital. Baba Farid University, Faridkot.

14. Abanti S, Das KN, Hazra A, Gharami CR, Chowdhury NS, et al. (2012) Cutaneous adverse drug reaction profile in a tertiary care outpatient setting in Eastern India. Indian Journal of Pharmacology 44: 792-797. 